\title{
Advances in early diagnosis of hepatocellular carcinoma
}

\author{
Partha Pratim Bose', Urmimala Chatterjee ${ }^{2}$ \\ ${ }^{1}$ Amity Institute of Applied Science, Amity University, Noida 201313, UP, India. \\ ${ }^{2}$ Delhi Public School, Howrah 711405, India.
}

Correspondence to: Dr. Partha Pratim Bose, Amity Institute of Applied Science, Amity University, Noida 201313, UP, India. E-mail:ppbose@amity.edu

How to cite this article: Bose PP, Chatterjee U. Advances in early diagnosis of hepatocellular carcinoma. Hepatoma Res 2019;5:24. http://dx.doi.org/10.20517/2394-5079.2019.10

Received: 9 Feb 2019 First Decision: 2 Apr 2019 Revised: 12 Apr 2019 Accepted: 18 Apr 2019 Published: 11 Jul 2019

Science Editor: Jin-Lin Hou Copy Editor: Cai-Hong Wang Production Editor: Jing Yu

\begin{abstract}
Hepatocellular carcinoma (HCC) is the most recurrent hepatic malignancy and the third in the cancer-related casualties in the west. The frequently-documented causes of $\mathrm{HCC}$ are chronic liver infections by hepatitis $\mathrm{B}$ virus or hepatitis $\mathrm{C}$ virus, nonalcoholic fatty liver disease, cirrhosis, exposure to aflatoxins and tobacco smocking, etc. Clinical presentation of this fatal disease ranges from asymptomatic to upper abdominal pain or common health conditions like weight loss or lethargy. Among current surveillance strategy for suspected patients, liver imaging and serum alpha fetoprotein estimation has been regularly recommended. However, sensitivity of this diagnostic methodology especially in early detections, often suffers from compromised sensitivity and selectivity. Various image based and serological biomarkers for HCC has been introduced in recent decades with varied sensitivity as stand-alone or combined diagnostic protocol. The current article will review the status of HCC diagnosis with respect to common diagnostic protocol, and upcoming novel biomarkers.
\end{abstract}

Keywords: Alpha fetoprotein, extracellular vehicles, hepatocellular carcinoma, indocyanine green, magnetic resonance imaging, non-coding RNA

\section{INTRODUCTION}

Hepatocellular carcinoma (HCC) is the most frequent primary liver malignancy and the third cause of cancerrelated death in the western countries that trended the highest increase in occurrence throughout the last decade $^{[1]}$. Patients with chronic HCV, hepatitis B (HBV), alcoholic liver disease and non-alcoholic fatty liver

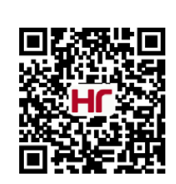


disease (NAFLD) often develop cirrhosis and are at high threat of developing HCC, with a significantly high annual incidence rates nearing $2 \%$ per year ${ }^{[2]}$. However, cirrhosis may or may not be present in HCC. Studies have revealed that NAFLD patients would be at a high risk of developing HCC, even in absence of cirrhotic transformation. The development of HCC is a complex multi-step process named as "hepatocarcinogenesis" and characterized by progressive genetic aberrations. Studies of these HCC associated molecular aberrations have also exposed that this malignancy is orchestrated by accumulation of some key genetic as well as epigenetic events which in turn lead to anomalous activation or inhibition of diverse cellular signaling cascades in crucial cellular processes like proliferation, cellular survival, differentiation, and angiogenesis. Further, the emergent views coming out of different studies suggest that the key cell biological events including regulation of p53/ARF, RB/INK4A and Wnt/ $\beta$-catenin pathways are also found to be affected in most cases of HCCs irrespective of etiology of the disease, indicating the presence of a shared oncogenic pathway in HCC development that regulates the mentioned biochemical events.

Clinical manifestation of HCC is diverse and significantly heterogeneous; asymptomatic cases are of suggestively high in incidence while symptoms often encompass from pain in upper right-abdominal quadrant, weight loss to obstructive jaundice and fatigue that are not strikingly exclusive compared to other hepatic ailments, viral infections, alcohol abuse associated functional loss of liver, etc. These make the differential detection, screening and monitoring of this fatal disease highly challenging.

Imaging is one of the gold standard non-invasive methods along with assessment of the biomarker alphafetoprotein (AFP) for diagnosis, staging and follow-up monitoring of HCC patients. Further, in standard clinical practice, AFP > $10 \mathrm{ng} / \mathrm{mL}$ or a composite AFP index ensures satisfactory sensitivity for early HCC $\operatorname{detection}^{[3]}$. However, there have been limitations often reported in sensitivity and specificity of AFP assessment and ultrasound based imaging with regard to clinical correlations with HCC. AFP-L3 and des- $\gamma$ carboxy prothrombin (DCP) are also used as important biomarkers for HCC determination ${ }^{[4]}$. HCC have a strong sex dominance in male ( 2 to 8 times more common) than in female in low - and high incidence areas. It has also been reported that the incidence of HCC holds a significant correlation with age, but a tendency of early onset of HCC in high-incidence areas has been observed too. With the continuous increase in incidence and mortality of HCC, along with the limitations of the current diagnostic strategy, the development of improved strategy for early detection is of greatest importance. The current review will discuss the latest clinical practice along with some recently introduced techniques in the early and sensitive diagnosis of HCC.

\section{METHODOLOGY}

\section{Clinical manifestation and image based analysis}

Often HCC progresses without prominent clinical symptoms and with no detectable aberration in liver function escaping early diagnosis. This makes HCC diagnosis usually delayed in developing countries with limited surveillance resources. On the other hand, clinical symptoms are emphasized in cases with compromised liver function. In advanced stages, symptoms include right upper quadrant abdominal pain, hepatomegaly, obstructive jaundice, hemobilia, and persistent fever. Some associated non-specific symptoms of malignant disease including anorexia, nausea, lethargy and weight loss are often reported. In many cases patients with unrecognized cirrhosis or known compensated cirrhosis may also present. Regular clinical practice employs various imaging studies such as ultrasonography (US), contrast enhanced computed tomography (CT) and magnetic resonance imaging (MRI) etc. for diagnosis, treatment management and follow up of HCC [Figure 1] ${ }^{[5]}$. According to the European Association for the Study of the Liver (EASL), liver lesions of length $2 \mathrm{~cm}$ or more observed on MRI or computed tomograph angiography (CTA), with AFP $>400 \mathrm{ng} / \mathrm{mL}$ or rising within sequential measurements do not require biopsy confirmation ${ }^{[6]}$. According to American Association for the Study of Liver Disease (AASLD) guideline however, liver lesions smaller 


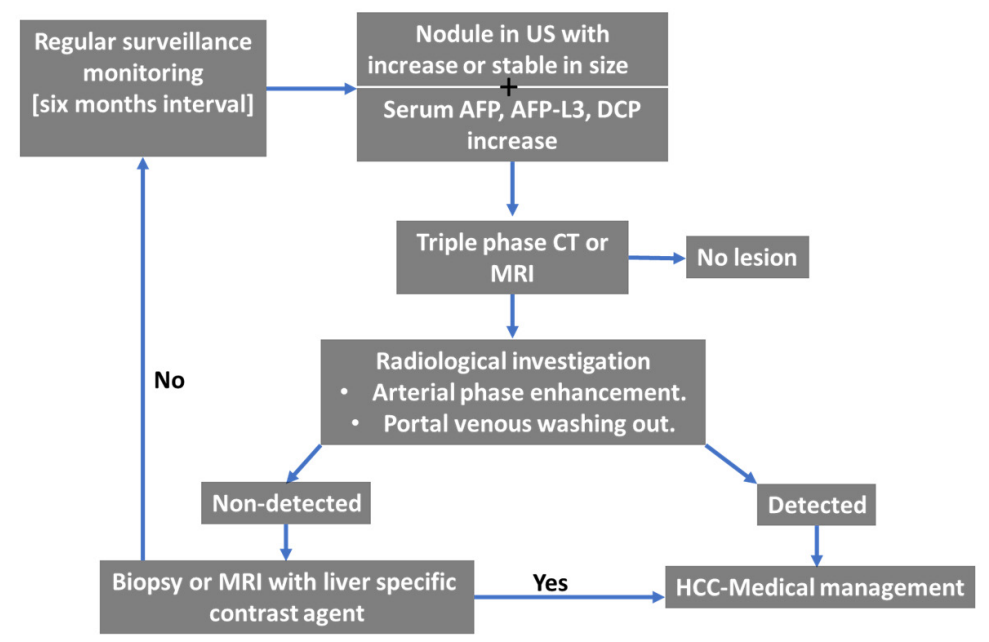

Figure 1. Current clinical practice for hepatocellular carcinoma (HCC) surveillance and diagnosis. AFP: alpha-fetoprotein; DCP: des- $\gamma$ carboxy prothrombin; MRI: magnetic resonance imaging

than even $1 \mathrm{~cm}$ has been recommended for re-examinations twice in a year and in case of no detectable radiographical changes over a period of two years, routine surveillance guideline should be followed ${ }^{[7]}$. In the case of non-cirrhotic patients vascular profiling of the liver tumor on imaging studies reveals no consistent clue for HCC, a biopsy of the lesion is recommended to rule out HCC. According to the recommendations of the Asia-Pacific Association for the Study of the Liver 2010, every nodular lesion with uncharacteristic vascular profiles should undergo further imaging investigation such as endoscopic ultrasonography (EUS) as the confirmation for $\mathrm{HCC}^{[8]}$. Contrast enhanced CT and MRI scans are most efficient in differentiate and analyze diverse liver nodules and can characterize late stage HCC by its exclusive appearance of arterialphase hypervascularity, however, this feature often lacks in early $\mathrm{HCC}^{[9,10]}$.

Diffusion weighted imaging (DWI) is one of the upcoming techniques that map the free diffusion of water molecules in three dimensions, which reflects the restricted diffusion in different stages of HCC and other liver diseases depending on highly congested cellularity, distortion of the extracellular interface, and density of the hydrophobic cell membrane within the tissue. This methodology has ability to detect liver lesions with quantitative investigation using no contrast media. Therefore, DWI can be used safely for the patients allergic to contrast media and the risk of nephrogenic complications associated with it can be avoided. However, in real life DWI is sensitive in detecting liver nodules, but it cannot precisely differentiate between HCC and dysplastic nodules or other malignant and benign lesions. Therefore, DWI results still require validations from contrast enhanced MRI for sensitive and specific determination of HCC cases ${ }^{[11]}$.

Indocyanine green (ICG) retention assay is another crucial fluorescence-based imaging technique that is being used for preoperative and perioperative dynamic diagnosis of HCC affected tissues. Both HCC affected tissue and normal tissue can take up ICG at same rate but follow up discharge of ICG from HCC tissues to the bile is impaired. There are few parallel phenomena that explain the reduced discharge rate of ICG form HCC tissues. Expression of glutathione S-transferase, an ICG binding protein, decreases significantly in HCC affected tissues than the healthy hepatocytes thus the excretion of ICG becomes impaired in cancer affected tissue of liver. Further, portal up-taking proteins like $\mathrm{Na}^{+} /$taurocholate co-transporting polypeptide (NTCP) and organic anion transporting polypeptide 8 (OATP8) are found to be overexpressed in case of HCC than the normal hepatocytes. Therefore, sustained portal intake of ICG by HCC by over expressed NTCP and OATP8 and impaired biliary excretion mechanism enhance the accumulation of ICG in HCC affected tissues that allows highly sensitive visualization of HCC affected part of liver following intravenous administration of $\mathrm{ICG}^{[2]}$. 


\section{Serological tests}

Serological biomarkers may provide crucial diagnostic hint in support of the results of ultrasound and may provide a crucial breakthrough in detecting biochemical changes related to liver malignancy prior to the image-based identification of hepatic nodules.

Assessment of the serum biomarker AFP has been one of the most extensively used clinical tests routinely performed for the determination of HCC. However, sensitivity of serum AFP test in determination of HCC ranges from $25 \%$ for nodules smaller than $3 \mathrm{~cm}$ to $50 \%$ for lesions larger than $3 \mathrm{~cm}$ in diameter ${ }^{[13]}$. Further, for the patients with cirrhosis of different stages AFP levels are found to be varying within a broad range and same trend has been found in the cases with underlying liver diseases where elevated serum AFP levels must be supported with high resolution image-based analysis to avoid false positive HCC detection ${ }^{[14]}$.

Other informative serological tests for HCC diagnosis are protein-induced by vitamin K absence or antagonistII (PIVKA-II), also known as DCP, and the percentage of Lens culinaris agglutinin-reactive alpha-fetoprotein (AFP-L3). Technically, AFP-L3 is a glycoform of AFP that exclusively originates from cancer cells and demonstrates higher specificity for HCC in combination with AFP ${ }^{[14]}$. However, AFP-L3 is not typically detected when AFP levels are $<20 \mathrm{ng} / \mathrm{mL}^{[8]}$ and it has a low sensitivity for early stage HCC diagnosis.

DCP or abnormal prothrombin cross-reacts with prothrombin antibodies in blood but fails to generate functional activity because it lacks $\gamma$-carboxy glutamate (GLA) unit which is crucial for binding $\mathrm{Ca}^{2+}$. Structural studies of HCC associated DCP revealed that it has only 5 GLA unit as compared to 10 GLA units in native prothrombin structure. The mean level of DCP in HCC patients were often found to be as high as $900 \mathrm{ng} / \mathrm{mL}$ when it was determined for the first time in a cohort of 76 patients; a significantly $67 \%$ of those patients had DCP levels above $300 \mathrm{ng} / \mathrm{mL}^{[15]}$. However, there are evidence that DCP sometimes may be present cases of hepatitis and metastatic carcinoma, with a lower level of less than $300 \mathrm{ng} / \mathrm{mL}$. Notably, plasma levels of abnormal prothrombin (DCP) in HCC could not be normalized by supplementing vitamin K, whereas, native prothrombin levels were recorded as normal. This rule out any correlation between HCC associated DCP production and vitamin $\mathrm{K}$ deficiency, but its biosynthesis in malignant hepatocyte is linked with an acquired defect in the vitamin K-dependent carboxylase system ${ }^{[16]}$. However, often AFP and DCP assay has found to be poorly correlated as it is expected from the very different origin of these biomarkers; the re-expression of a fetal antigen in the tumor tissues and an independently acquired posttranslational aberration respectively. DCP measured in biopsy homogenates of HCCs showed a high upward trend compared to the plasma concentration of the corresponding patients. Notably, in patients having normal plasma levels of DCP showed no changes in DCP concentrations within biopsy homogenates and surrounding healthy hepatic tissues. Currently, plasma DCP levels greater than $100 \mathrm{ng} / \mathrm{mL}$ on ELISA are taken as suggestive of HCC ${ }^{[17]}$. However, DCP levels and tumor size do not correlate well, studies found that DCP levels increased in only $20 \%$ of the HCC cases with nodules less than $3 \mathrm{~cm}^{[18]}$. A recent French study adopting a lower cut-off of DCP $(42 \mathrm{ng} / \mathrm{mL}$ ) recorded a sensitivity and specificity of $77 \%$ and $82 \%$, respectively for early diagnosis of HCC; however, less sensitivity and specificity ( $61 \%$ and $50 \%$ respectively) were registered with a lower AFP cut-off of $5.5 \mathrm{ng} / \mathrm{mL}^{[19]}$. A recent study demonstrated that DCP was superior to AFP or its variant biomarker, AFP-L3 in detecting HCC and a combination of DCP with the other two mentioned tests provided in better accuracy than DCP alone ${ }^{[20]}$.

In current clinical practice, diagnostic methodology and surveillance program for HCC follow a sequence of image based and serological tests as described in Figure 1. Under clinical guideline of AASLD, the European Association for the Study of the Liver and the European Organization for Research and Treatment of Cancer (EASL-EORTC), and the Japan Society of Hepatology (JSH), those with cirrhosis and those with chronic HBV infection regardless of cirrhosis has been considered as the high-risk population for HCC surveillance. However, EASL-EORTC also includes patients with chronic HCV and advanced liver fibrosis in this high- 
risk group. In a real clinical surveillance protocol a combination of ultrasound based analysis and serological determination of biomarkers: AFP, AFP-L3 and DCP increases the diagnostic accuracy to a significant level compared to only image based study. However, ultrasound is reported to be precision compromised in visualizing the liver in patients with morbid obesity, therefore, in next level of confirmation triple phase CT or MRI is recommended. However, ultrasound positive, MRI negative cases are further cross-checked and confirmed either by biopsy or MRI with liver specific contrast agent under this HCC surveillance guidance. Based on 85-171 days of median doubling time in HCC volume, a 6-month interval in the surveillance protocol is currently recommended. However, the JSH guidelines propose a 3-4 month interval for HBV and HCV associated liver cirrhosis patients.

The widely approved strategy for the surveillance of HCC as mentioned above is a combination of image based and serological analysis with varied stand alone and combined sensitivity and diagnostic accuracy which provide impetus for the clinical assessment of new biomarker for HCC.

A recent study on the diagnostic performance of serum aldo-keto reductase family 1 member B10 (AKR1B10) in hepatitis B virus/hepatitis $\mathrm{C}$ virus (HBV/HCV)-related liver diseases demonstrated a crucial correlation in detection of HCC. Significant and stage dependent elevation of serum levels of AKR1B10 were recorded in patients with HCC compared to liver disorder cases. Importantly, comparison of advanced and terminal of HCC cases, a crucial increase in AKR1B10 levels was reported in early and intermediate HCC stages. The reported sensitivity $(81.0 \%)$ and specificity $(60.9 \%)$ for AKR1B10 based HCC diagnosis were significantly high at a cutoff value of $1.51 \mathrm{ng} / \mathrm{mL}$. Further, conjoint measurement of serum AKR1B10 and AFP significantly increased sensitivity and specificity of the combined diagnostic parameters ${ }^{[2,22]}$.

Cell secreted small membrane-enclosed spheres, present in biological fluids are known as extracellular vehicles (EVs). EVs contain diverse types of biomolecules, including proteins, RNA, DNA, various metabolites and lipids, etc. that often carries the signature of the ailing conditions of the tissues of their origin. Thus, EVs are often potential source of biomarkers for different human pathobiology. Several lines of different diagnostic reports suggested that HCC cell-derived EVs carries the key effectors for autocrine and/ or paracrine cellular communications, chemoresistance, angiogenesis, and tumor dissemination. A study has been successful in identifying EVs secreted by sorafenib-treated HCC cells rich in long intergenic noncoding RNA regulator for reprogramming (linc-ROR) that enables them to escape chemotherapy-induced apoptosis by the mechanism of p53 repression, upregulating the expression of CD133 marker and the stimulation of the signaling pathway for hepatocyte growth factor (HGF)/c-Met/Akt in liver cancer cells ${ }^{[23]}$. The notable abundance of EVs in biological fluids and their varied molecular payload has recently upgraded EVs as a key source of non-invasive biomarkers in liver diseases. Diagnostic studies have demonstrated the significantly elevated levels of different micro-RNAs in serum EVs of HCC patients (miRs 18a, 221, 222, and 224) as compared to cirrhosis or HCB patients, whereas a prominent drop in the concentration of miR-21 was consistently observed in the serum EVs of HCC patients as compared to HBV or healthy controls ${ }^{[24]}$. Another important molecular cargo of EVs isolated from HCC has been miR-665, whose concentration has found to be positively correlated with tumor size, and clinical stages along with local invasion ${ }^{[25]}$. Reduced expression of another EV cargo, miR-718 is highly correlated with increasing tumor size, recurrence and poor histological differentiation of HCC cells ${ }^{[26]}$. Furthermore, low concentrations of serum EV packed miR$125 \mathrm{~b}$ have found to be associated to advanced TNM staging parameters, which is a suggestive of miR-125b as a potential prognostic biomarker for recurrence and overall survival of $\mathrm{HCC}^{[27]}$. Further, recent studies suggest that apart from miRNAs, serum EVs has been the delivery mode of diverse set of HCC associated proteins such as LG3BP, polymeric immunoglobulin receptor (PIGR) and alpha-2-macroglobulin (A2MG). These proteins were found to be over expressed in HCC patients compared to healthy individuals and serum EV concentrations of these proteins provided even better diagnostic value than AFP in early diagnosis of $\mathrm{HCC}^{[28]}$. Serum EV concentration itself is an implicative of HCC progression; it has been demonstrated that stage I and II HCC patients recorded higher serum EV abundance compared to liver cirrhosis patients ${ }^{[29]}$. 
Table 1. Aberrant concentration of miRNAs observed in hepatocellular carcinoma patients (with hepatitis B virus infection)

\begin{tabular}{lll}
\hline miRNA & Change in concentration & Isolated sample \\
\hline miR-221 & Upregulation & Tissue and Serum \\
miR-21 & Upregulation & Tissue and Serum \\
miR-222 & Upregulation & Tissue and Serum \\
miR-222a & Upregulation & Serum \\
miR-224 & Upregulation & Tissue and Serum \\
miR-101 & Downregulation & Tissue \\
miR-18a & Upregulation & Tissue and Serum \\
miR-223 & Upregulation & Serum \\
\hline
\end{tabular}

Table 2. Aberrant concentration of miRNAs observed in hepatocellular carcinoma patients (with hepatitis C virus infection)

\begin{tabular}{lcl}
\hline miRNA & Change in concentration & Isolated sample \\
\hline miR-765 & Upregulation & Urine \\
miR-200a & Upregulation & Urine \\
miR-610 & Upregulation & Urine \\
miR-323 & Downregulation & Urine \\
miR-449 & Downregulation & Urine \\
miR-502d & Downregulation & Urine \\
miR-92b & Downregulation & Urine \\
miR-122 and miR-221 & Upregulation & Serum \\
miR-181a & Upregulation & Tissue and PBMC \\
miR-9, -10a, $-15 a,-16$ & Upregulation & Tissue \\
miR-198, -302b, $-145,-368,-218,-330,-137,-147$ & Downregulation & Tissue from primary liver tumor \\
miR-155 & Upregulation & Tissue \\
\hline
\end{tabular}

Recent study suggests that a significant number of non-coding RNAs (miRNA and long non-coded RNA; lncRNA) have been associated with HCC, more precisely that caused by HCV infection. These noncoding RNAs are found to be differentially expressed to promote pathogenesis of HBV and HCV-induced HCC. Apart from miRNAs packed in EVs (as discussed above); serum, urine, tissue concentration of various miRNAs also afford promise to be potential future biomarkers for both HBV or HCV-induced HCC as they are correlated consistently with progression, staging, survival rate and recurrence [Tables 1 and 2$]^{[30]}$.

Many of the LncRNAs (long noncoding RNAs) are found to be dysregulated significantly in HCC and most are associated with the maintenance of the pathophysiological ambience of HCC tissue [Table 3]. The recent trend is also suggestive of their potential use as future biomarker candidates for HCC diagnosis. A decisive upregulation is observed in a lncRNA, HULC (highly upregulated in liver cancer) with a significantly consistent correlation with HCC progression, hepatic colorectal metastasis in HCC etc. Further, a key single nucleotide polymorphism in HULC (SNP) has been identified in the serum sample that can be a susceptibility marker for the risk of HBV infection ${ }^{[31,32]}$. H19 is another crucial lncRNA highly expressed in fetal liver, faintly expressed in normal adult liver. However, during tumorigenesis, it is highly upregulated and expressed, and might play a crucial part in tumorigenesis. Further, H19 was found to be over expressed and associated with cell proliferation, invasion, chemoresistance in HCC and thus it holds the promise of being a potential future serum biomarker for the same ${ }^{[33]}$. Among other lncRNAs, HOTAIR, MALAT-1, MEG3, GAS5, UCA1, HOTTIP, XIST are found to be consistently dysregulated in HCC and have the potential to act as crucial HCC biomarkers in future if validated by different cohorts of clinical data ${ }^{[34-40]}$.

A recent study demonstrated that a secretory protein, Trefoil factor 3 (TFF3), was highly expressed in HCC tissues, suggesting it to be a potential serum biomarker for HCC. Further, two circulating microRNAs (miR-7-5p and miR-203a-3p) reported to target TFF3 have also been proposed as future biomarkers for HCC. However exhaustive clinical data are awaited ${ }^{[41]}$. 
Table 3. Dysregulated IncRNAs observed in hepatocellular carcinoma

\begin{tabular}{|c|c|c|}
\hline IncRNA & Change in concentration & Isolated sample \\
\hline HULC & Upregulation & Hepatic colorectal metastasis samples \\
\hline $\mathrm{H} 19$ & Upregulation & Malignant liver tissues \\
\hline UCA1 & Upregulation & Liver tissues and serum \\
\hline HOTAIR & Upregulation & Malignant liver tissues \\
\hline MVIH & Upregulation & Malignant liver tissues \\
\hline ATB & Upregulation & Malignant liver tissues \\
\hline HOTTIP & Upregulation & Malignant liver tissues \\
\hline MALAT-1 & Upregulation & Malignant liver tissues \\
\hline VLDLR & Upregulation & Malignant liver tissues and EVs \\
\hline TUC339 & Upregulation & EV \\
\hline MEG3 & Downregulation & Malignant liver tissues \\
\hline PTENP1 & Downregulation & Malignant liver tissues \\
\hline DREH & Downregulation & Malignant liver tissues \\
\hline WT1-AS & Downregulation & Malignant liver tissues \\
\hline Uc002mbe.2 & Downregulation & Malignant liver tissues \\
\hline XIST and FTX & Downregulation & Expressed more in liver malignant tissues of female than male \\
\hline CPS1-IT1 & Downregulation & Malignant liver tissues \\
\hline $\mathrm{AOC}-4 \mathrm{P}$ & Downregulation & Malignant liver tissues \\
\hline $\mathrm{HEIH}$ & Upregulation & Malignant liver tissues \\
\hline
\end{tabular}

Recently, among various non-protein biomarkers, aberrant methylation, like hypomethylation in DNA and/ or hypermethylation in the $\mathrm{CpG}$ promoter gene, are found to be associated in the pathogenesis of different tumors, including liver $\mathrm{HCC}^{[42,43]}$. A recent study identified a set of aberrantly methylated DNA markers showing significant association with the HCC progression and interestingly, most of these hypermethylation occur within the $\mathrm{CpG}$ domain ${ }^{[4]}$. The identification of these circulating tumor DNAs carrying the hypermethylated aberration within in the large cohort of HCC patients provides a promise of development of a highly sensitive, noninvasive and accurate early detection platform for HCC.

\section{CONCLUSION}

HCC remains to be the most fatal malignant liver cancer worldwide even after the advances that has been achieved in diagnostic and invasive medicine since last decades. The contemporary HCC treatment has been intensive to early diagnosis and hepatic transplantation as medical management of this fatal disease. Combination therapies performs well to downgrade the tumor and make it removable, that significantly improve basic liver function and improve the timeline of survival, however, the early diagnosis has been the most crucial deciding factor. In summary, informative feedback on the preclinical performance of imagebased techniques, AFP, AFP-L 3\%, and DCP in the detection of HCC has created a vast and varied data set since last few decades that serves as a fulcrum in the early diagnosis and medical management of HCC. Recent observations demonstrate that many miRNAs and lncRNAs are differentially expressed in malignant liver tissues, and their dysregulation as reflected in aberrant concentrations in various clinical samples (tissues, serum, EVs etc.) were found to be correlated well with HCC progression, recurrence after liver transplantation, chemoresistance etc. Thus, these miRNAs or lncRNAs may serve as potential biomarkers for the diagnosis, prognosis, prediction of recurrence and therapeutic response of $\mathrm{HCC}^{[45]}$.

However, a substantial heterogeneity among various cohorts of patients in terms of diagnostic criteria leaves the space for evaluating the clinical performance of novel biomarkers, comprehensive studying different variables associated with the malignant transformation in HCC and inclusion of some of the newer biomarkers in surveillance strategy may prove to be crucial in future clinical management of this fatal disease. 


\section{DECLARATIONS}

\section{Authors' contributions}

Literature survey; Data analysis and comparison of published data; Draft of the manuscript: Bose PP Draft of the Manuscript and comparison of published data: Chatterjee $U$

\section{Availability of data and materials}

Not applicable.

\section{Financial support and sponsorship}

Not applicable.

\section{Conflicts of interest}

All authors declared that there are no conflicts of interest.

\section{Ethical approval and consent to participate}

Not applicable.

\section{Consent for publication}

Not applicable.

\section{Copyright}

(c) The Author(s) 2019.

\section{REFERENCE}

1. Massarweh NN, El-Serag HB. Epidemiology of hepatocellular carcinoma and intrahepatic cholangiocarcinoma. Cancer Control 2017;24:1073274817729245.

2. Howe HL, Wu X, Ries LA, Cokkinides V, Ahmed F, et al. Annual report to the nation on the status of cancer, 1975-2003, featuring cancer among U.S. Hispanic/Latino populations. Cancer 2006;107:1711-42.

3. Kanwal F, Kramer JR, Mapakshi S, Natarajan Y, Chayanupatkul M, et al. Risk of hepatocellular cancer in patients with non-alcoholic fatty liver disease. Gastroenterology 2018;155:1828-37.e2.

4. Marrero JA, Feng Z, Wang Y, Nguyen MH, Befeler AS, et al, Alpha-fetoprotein, des- $\gamma$ carboxyprothrombin, and lectin-bound alphafetoprotein in early hepatocellular carcinoma. Gastroenterology 2009;137:110-8.

5. Ghanaati H, Alavian SM, Jafarian A, Ebrahimi Daryani N, Nassiri-Toosi M. Imaging and imaging-guided interventions in the diagnosis and management of hepatocellular carcinoma (HCC)-review of evidence. Iran J Radiol 2012;9:167-77.

6. Haug AR.. Comparison of liver biopsy and noninvasive methods for diagnosis of hepatocellular carcinoma Imaging of primary liver tumors with positron-emission tomography. Q J Nucl Med Mol Imaging 2017;61:292-300.

7. Omata M, Lesmana LA, Tateishi R, Chen PJ, Lin SM, et al. Asian pacific association for the study of the liver consensus recommendations on hepatocellular carcinoma. Hepatol Int 2010;4:439-74.

8. Navin PJ, Venkatesh SK. Hepatocellular carcinoma: state of the art imaging and recent advances. J Clin Transl Hepatol 2019;7:72-85.

9. Jang HJ, Kim TK, Burns PN, Wilson SR. Enhancement patterns of hepatocellular carcinoma at contrast-enhanced US: comparison with histologic differentiation. Radiology 2007;244:898-906.

10. Wu B, Zhang Y, Tan H, Shi H. Value of ${ }^{18} \mathrm{~F}-\mathrm{FDG}$ PET/CT in the diagnosis of portal vein tumor thrombus in patients with hepatocellular carcinoma. Abdom Radiol (NY) 2019; doi: 10.1007/s00261-019-01997-2.

11. Park MS, Kim S, Patel J, Hajdu CH, Do RK, et al. Hepatocellular carcinoma: detection with diffusion weighted versus contrastenhanced magnetic resonance imaging in pretransplant patients. Hepatology 2012;56:140-8.

12. Ishizawa T, Masuda K, Urano Y, Kawaguchi Y, Satou S, et al. Mechanistic background and clinical applications of indocyanine green fluorescence imaging of hepatocellular carcinoma. Ann Surg Oncol 2014;21:440-8.

13. Hester CA, Rich NE, Singal AG, Yopp AC. Comparative analysis of nonalcoholic steatohepatitis versus viral hepatitis and alcoholrelated liver disease-related hepatocellular carcinoma. J Natl Compr Canc Netw 2019;17:322-9.

14. Li D, Mallory T, Satomura S. AFP L3: a new generation of tumor marker for hepatocellular carcinoma. Clin Chim Acta 2001;313:15-9.

15. Liebman HA, Furie BC, Tong M, Blanchard RA, Lo KJ, et al. Des- $\gamma$-carboxy (abnormal) prothrombin as a serum marker of primary hepatocellular carcinoma. N Engl J Med 1984;310:1427-31.

16. Wang B, Zhu J, Ma X, Wang H, Qiu S, et al. Platelet activation status in the diagnosis and postoperative prognosis of hepatocellular carcinoma. Clin Chim Acta 2019;495:191-7. 
17. Ono M, Ohta H, Ohhira M, Sekiya C, Namiki M. Measurements of immunoreative prothrombin, des- $\gamma$-carboxy prothrombin and vitamin $\mathrm{K}$ in human liver tissues: overproduction of immunoreactive prothrombin in hepatocellular carcinoma. Am J Gastroenterol 1990;85:1149-54.

18. Tsai SL, Huang GT, Yang PM, Sheu JC, Sung JL, et al. Plasma des-gamma-carboxyprothrombin in the early stages of hepatocellular carcinoma. Hepatology 1990;11:481-8.

19. Pote N, Cauchy F, Albuquerque M, Voitot H, Belghiti J, et al. Performance of PIVKA-II for early hepatocellular carcinoma diagnosis and prediction of microvascular invasion. J Hepatol 2015;62:848-54.

20. Lim TS, Kim DY, Han KH, Kim HS, Shin SH, et al. Combined use of AFP, PIVKA-II, and AFP-L3 as tumor markers enhances diagnostic accuracy for hepatocellular carcinoma in cirrhotic patients. Scand J Gastroenterol 2016;51:344-53.

21. Han C, Gao L, Bai H, Dou X. Identification of a role for serum aldoketo reductase family 1-member B10 in early detection of hepatocellular carcinoma. Oncology Letters 2018;16:7123-30.

22. DiStefano JK, Davis B. Diagnostic and prognostic potential of AKR1B10 in human hepatocellular carcinoma. Cancers (Basel) 2019;11:E486.

23. Conigliaro A, Costa V, Lo Dico A, Saieva L, Buccheri S, et al. CD90+ liver cancer cells modulate endothelial cell phenotype through the release of exosomes containing H19 lncRNA. Mol Cancer 2015;14:155.

24. Wang H, Hou L, Li A, Duan Y, Gao H, et al. Expression of serum exosomal microRNA-21 in human hepatocellular carcinoma. Biomed Res Int 2014;2014:864894.

25. Qu Z, Wu J, Wu J, Ji A, Qiang G, et al. Exosomal miR-665 as a novel minimally invasive biomarker for hepatocellular carcinoma diagnosis and prognosis. Oncotarget 2017;8:80666-78.

26. Sugimachi K, Matsumura T, Hirata H, Uchi R, Ueda M, et al. Identification of a bona fide microRNA biomarker in serum exosomes that predicts hepatocellular carcinoma recurrence after liver transplantation. Br J Cancer 2015;112:532-8.

27. Liu W, Hu J, Zhou K, Chen F, Wang Z, et al. Serum exosomal miR-125b is a novel prognostic marker for hepatocellular carcinoma. OncoTargets Ther 2017;10:3843-51.

28. Arbelaiz A, AzkargortaM, KrawczykM, Santos-Laso A, Lapitz A, et al. Serum extracellular vesicles contain protein biomarkers for primary sclerosing cholangitis and cholangiocarcinoma. Hepatology 2017;66:1125-43.

29. Wang W, Li H, Zhou Y, Jie S. Peripheral blood microvesicles are potential biomarkers for hepatocellular carcinoma. Cancer Biomark 2013;13:351-7.

30. Świtlik WZ, Bielecka-Kowalska A, Karbownik MS, Kordek R, Jabłkowski M, et al. Forms of diagnostic material as sources of miRNA biomarkers in hepatocellular carcinoma: a preliminary study. Biomark Med 2019; doi: 10.2217/bmm-2018-0485.

31. Panzitt K, Tschernatsch MM, Guelly C, Moustafa T, Stradner M, et al. Characterization of HULC, a novel gene with striking upregulation in hepatocellular carcinoma, as noncoding RNA. Gastroenterology 2007;132:330-42.

32. Liu Y, Pan S, Liu L, Zhai X, Liu J, et al. A genetic variant in long non-coding RNA HULC contributes to risk of HBV-related hepatocellular carcinoma in a Chinese population, PLoS One 2012;7:e35145.

33. Smaldone MC, Davies BJ. BC-819, a plasmid comprising the H19 gene regulatory sequences and diphtheria toxin A, for the potential targeted therapy of cancers. Curr Opin Mol Ther 2010;12:607-16.

34. Yang L, Zhang X, Li H, Liu J. The long noncoding RNA HOTAIR activates autophagy by upregulating ATG3 and ATG7 in hepatocellular carcinoma. Mol Biosyst 2016;12:2605-12.

35. Lai MC, Yang Z, Zhou L, Zhu QQ, Xie HY, et al. Long non-coding RNA MALAT-1 overexpression predicts tumor recurrence of hepatocellular carcinoma after liver transplantation. Med Oncol 2012;29:1810-6.

36. Braconi C, Kogure T, Valeri N, Huang N, Nuovo G, et al. MicroRNA-29 can regulate expression of the long non-coding RNA gene MEG3 in hepatocellular cancer. Oncogene 2011;30:4750-6.

37. Tu ZQ, Li RJ, Mei JZ, Li XH. Down-regulation of long non-coding RNA GAS5 is associated with the prognosis of hepatocellular carcinoma. Int J Clin Exp Pathol 2014;7:4303-9.

38. Wang F, Ying HQ, He BS, Pan YQ, Deng QW, et al. Upregulated lncRNA-UCA1 contributes to progression of hepatocellular carcinoma through inhibition of miR-216b and activation of FGFR1/ERK signaling pathway. Oncotarget 2015;6:7899-917.

39. Tsang FH, Au SL, Wei L, Fan DN, Lee JM, et al. Long non-coding RNA HOTTIP is frequently up-regulated in hepatocellular carcinoma and is targeted by tumour suppressive miR-125b. Liver Int 2015;35:1597-606.

40. Zhuang LK, Yang YT, Ma X, Han B, Wang ZS, et al. MicroRNA-92b promotes hepatocellular carcinoma progression by targeting Smad7 and is mediated by long non-coding RNA XIST. Cell Death Dis 2016;7:e2203.

41. Zhang C, Xia R, Zhang B, Wang H. The predictive powers of plasma trefoil factor 3 or its related micro RNAs for patients with hepatocellular carcinoma. BMC Cancer 2018;18:1110.

42. Ezzat WM, Amr KS, Elhosary YA, Hegazy AE, Fahim HH, et al. Detection of DNA methylated microRNAs in hepatocellular carcinoma. Gene 2019;702:153-7.

43. Xu RH, Wei W, Krawczyk M, Wang W, Luo H, et al. Circulating tumour DNA methylation markers for diagnosis and prognosis of hepatocellular carcinoma. Nat Mater 2017;16:1155-61.

44. Kisiel JB, Dukek BA, Kanipakam RVSR, Ghoz HM et al. Hepatocellular carcinoma detection by plasma methylated DNA: discovery, phase I pilot, and phase II clinical validation. Hepatology 2019;69:1180-92.

45. Li Y, Zhao J, Yu S, Wang Z, He X, et al. Extracellular Vesicles Long RNA Sequencing Reveals Abundant mRNA, circRNA, and lncRNA in Human Blood as Potential Biomarkers for Cancer Diagnosis. Clin Chem 2019; doi: 10.1373/clinchem.2018.301291. 